\section{Next level immediacy}

As part of its comprehensive ImmediaXy suite of solutions, Straumann takes immediacy to the next level. It offers an ecosystem of solutions designed to facilitate efficient and confident immediate temporisation for single units, multiple teeth or full-arch cases.

This includes:

- 3Shape Trios intraoral scanner for accurate data capture

- Straumann DiagnostiX and Straumann Cares Visual Chairside software for streamlined surgical and prosthetic planning

- Straumann BLX implant with innovative SLActive surface for high primary stability and predictability of immediate loading protocols

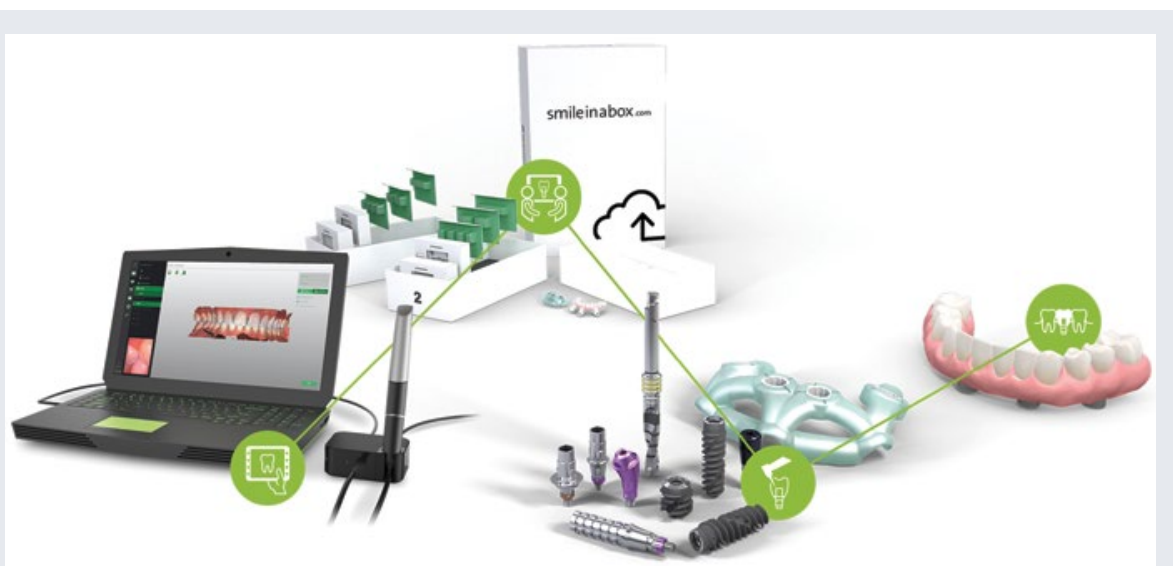

- Straumann Cares C Series for chairside milling of temporary restorations.

Straumann has been a leader in the global dental implant market for several years now, having established a reputation for cutting-edge solutions backed by science.
Its ImmediaXy solutions are a must for any practitioner looking to optimise predictability, flexibility and longevity of their immediate implant treatments.

For more information about the Straumann ImmediaXy suite of solutions, visit www.straumann.com.

\title{
All-in-one and all-round protection
}

Last year Dentsply Sirona launched its new DAC Universal with a modern look and numerous changes based on the needs of the customers, eg Touch-Display, LAN interface, guided Check \& Clean. This year the new DAC Universal $S$ is ready to take things even further. The beneficial features of the DAC Universal are continued in the DAC Universal S and now supplemented with a complete sterilisation function - especially for markets that require sterilisation instead of a disinfection of unwrapped medical devices.

With two programmes to choose from the automatic programme selection of the DAC Universal $S$ continues to provide maximum process reliability: the Pink Lid Program is specially designed for rotating instruments such as turbines, straight and contra-angle handpieces. The reprocessing process starts with a cold pre-wash. After an internal cleaning of the spray and gear channels a fully automatic lubrication follows. External cleaning with the pulse-wash method removes dirt and deposits from the outer surfaces of the instruments. The cleaning performance is excellent: the results far exceed the standard values ( $80 \%$ better) required by the Robert Koch-Institute. Finally, instruments are sterilised at $134^{\circ} \mathrm{C}$ with three minutes holding time, and then cooled with cold air. The second programme, the White Lid Program, is used for instruments that do not require lubrication: eg ultrasonic handpieces and tips or nozzles of multifunctional syringes and powder jet devices as well as powder jet handpieces. The cleaning steps are almost identical to the Pink Lid Program, but with lubrication step removed.

The DAC Universal S uses only demineralised water for all cleaning and sterilisation steps, and does not require chemical cleaning agents, therefore preserving the lifespan of instruments. The guided maintenance workflow Check \& Clean and the LAN interface for electronic documentation systems are also integrated into the DAC Universal S.

The complete process takes approximately 21 minutes for each programme - a fully compliant hygiene cycle with just a press of a single button.

Visit www.dentsplysirona.com for more information about Dentsply Sirona and its products.

\section{PPE with same day dispatch}

In these challenging times, Kent Express will support you with great value across its huge range of PPE, including FFP2 and FFP3 respirator masks, Type IIR and Type II surgical masks, gowns, visors, gloves and more. Together with their comprehensive range of over 25,000 dental products, Kent Express stocks everything you need to keep your practice running effectively. Order online by $5 \mathrm{pm}$ for free same day dispatch plus next day delivery.
As a trusted supplier of PPE and other dental supplies for over 35 years, Kent Express' aim is to make your life easier through their expertise, partnership and service. For example, regular customers have the opportunity to fix their buying prices for six months - saving you money, and helping with forward planning.

Currently you can save up to $30 \%$ off list price on a wide range of products in Kent Express' 'Return to Practice' offer, including hand instruments, bonding, cements and

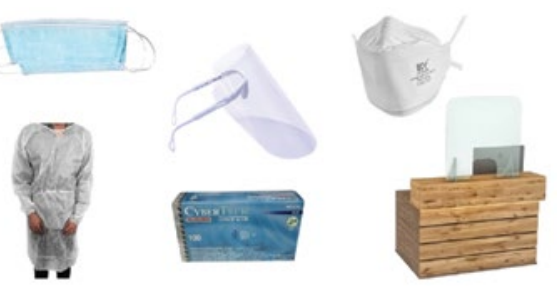

liners, infection control, restoratives and small equipment.

Visit kentexpress.co.uk to order now, or call the experienced team on 0800028 1181 (Mon-Fri $8.30 \mathrm{am}-5.30$ pm). 